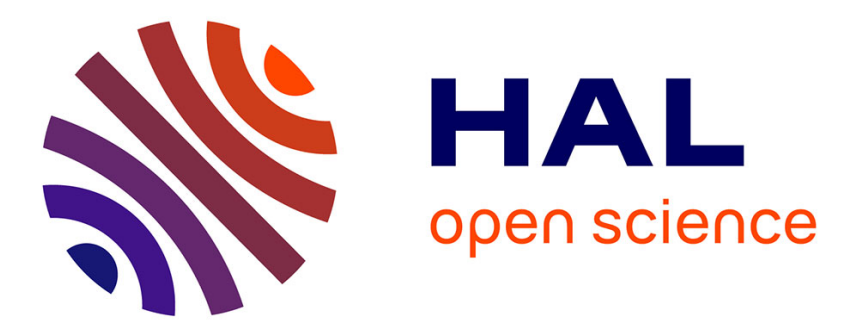

\title{
Une tumeur rénale pas si conventionnelle...
}

S. El Naderi, C. Sader Ghorra, R. Abou-Jaoudé, F. Llamas-Gutierrez, N.

Rioux-Leclercq

\section{To cite this version:}

S. El Naderi, C. Sader Ghorra, R. Abou-Jaoudé, F. Llamas-Gutierrez, N. Rioux-Leclercq. Une tumeur rénale pas si conventionnelle.... Annales de Pathologie, A paraître, 10.1016/j.annpat.2020.02.028. hal-02865293

\section{HAL Id: hal-02865293 \\ https://hal-univ-rennes1.archives-ouvertes.fr/hal-02865293}

Submitted on 17 Jun 2020

HAL is a multi-disciplinary open access archive for the deposit and dissemination of scientific research documents, whether they are published or not. The documents may come from teaching and research institutions in France or abroad, or from public or private research centers.
L'archive ouverte pluridisciplinaire HAL, est destinée au dépôt et à la diffusion de documents scientifiques de niveau recherche, publiés ou non, émanant des établissements d'enseignement et de recherche français ou étrangers, des laboratoires publics ou privés. 
Titre: Une tumeur rénale pas si conventionnelle...

Title: An unconventional renal tumor...

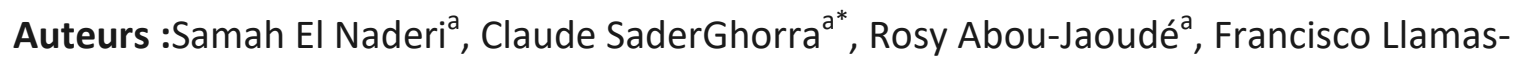
Gutierrez $^{\mathrm{b}}$, Nathalie Rioux-Leclerq ${ }^{\mathrm{b}}$

Affiliations et coordonnées:

${ }^{a}$ Service d'anatomie et cytologie pathologiques, Centre Hospitalier Universitaire Hôtel-Dieu de France, Université Saint-Joseph, Beyrouth, Liban

${ }^{b}$ Service d'anatomie et cytologie pathologiques, Centre Hospitalier Universitaire de Rennes, 35033 Rennes cedex 9, France

*Auteur correspondant : adresse e-mail : cghorra18@gmail.com (Claude SaderGhorra)

Mots clés : Rein ; carcinome rénal ; lymphome rénal

Keywords: Kidney; renal carcinoma; renal lymphoma

\section{Observation}

Un homme de 61 ans sans antécédents notables, s'est présenté pour une altération de l'état général et une perte de poids de $3 \mathrm{~kg}$ en un mois. L'examen physique et le bilan biologique étaient sans particularités. L'examen tomodensitométrique et I'IRM abdomino-pelviens ont révélé une masse de $4 \mathrm{~cm}$ environ du rein gauche. Les ganglions rétro-péritonéaux et à distance étaient normaux. Le patient a été opéré d'une néphrectomie radicale gauche.

Le rein comportait une masse centro-hilaire mesurant 3,7 x 3,2 cm, de couleur brun chamois, montrant des modifications kystiques. Cette tumeur était adhérente à la graisse péri-rénale, sans signe d'envahissement des cavités pyélocalicielles ni du sinus rénal.

L'examen microscopique a montré une tumeur bien circonscrite faite de deux composantes intimement intriquées (Fig. 1). La première composante, de loin prédominante, était de nature épithéliale, et présentait une architecture essentiellement tubuleuse. Les cellules tumorales avaient des cytoplasmes abondants clairs, et des noyaux irréguliers avec des nucléoles visibles au grossissement $\times 40$ (Grade 2 de Führman). Ces cellules étaient positives pour la Pancytokératine AE1/AE3, la CK7, la Vimentine, le PAX8, et négatives pour le CD10, le RCC, la CAIX et le TFE3 (Fig. 2).

L'œil était ensuite attiré, initialement au faible grossissement, au sein du stroma, par une population monotone, diffuse, faite de nappes de cellules isolées de grande taille. Au plus fort grossissement, cette prolifération cellulaire d'allure lymphomateuse était composée de nappes de cellules d'aspect centroblastique, aux cytoplasmes peu abondants et aux noyaux volumineux, renfermant un à plusieurs nucléoles proéminents. De nombreuses mitoses étaient retrouvées. En immunohistochimie, cette composante, négative aux cytokératines, avait montré une positivité aux immunomarquages suivants : $C D 20, b c l-2, b c l-6, M U M 1$, et un indice de prolifération atteignant les $90 \%$. Elle était négative pour le CD5, le CD10, le CD23 et c-MYC (Fig. 3). Cette composante lymphomateuse à grandes cellules B est de profil immunohistochimique non-GC, selon l'algorithme de Hans. L'analyse par technique de FISH à la 
recherche d'une trisomie 7, d'une trisomie 17, d'un réarrangement TFE3 et d'une délétion VHL était négative ainsi que I'hybridation in situ EBER.

\section{Quel est votre diagnostic ? \\ Diagnostic}

Une tumeur rénale hybride associant un carcinome rénal à cellules claires, et un lymphome B diffus à grandes cellules de profil non centro-germinatif.

\section{Discussion}

Le lymphome rénal primitif est une tumeur rare, correspondant à moins de $1 \%$ de tous les lymphomes extra-ganglionnaires, avec seulement une cinquantaine de cas rapportés dans la littérature. Par définition, il s'agit d'un lymphome non hodgkinien survenant au sein du parenchyme rénal, sans évidence d'atteinte ganglionnaire, et avec un bilan d'extension négatif. II s'agit le plus souvent d'un lymphome B diffus à grandes cellules[1,2]. Le carcinome rénal à cellules claires (CRCC) représente quant à lui à $65-70 \%$ de tous les cancers du rein. La revue de la littérature se focalise surtout sur la survenue de façon secondaire d'un carcinome rénal chez les patients ayant été traité pour un lymphome. Ce phénomène pourrait être expliqué par les effets tardifs du traitement, par la survie prolongée de ces patients souvent immunocompromis, et par des facteurs biologiques et génétiques qui ne sont pas encore entièrement élucidés[3,4]. Cependant, la survenue synchrone du lymphome rénal primitif et du CRCC est un phénomène très inhabituel, avec seulement un cas décrit dans la littérature[5].

Le processus étiopathogénique pouvant expliquer l'émergence d'un lymphome rénal primitif reste toujours non élucidé. Un développement à partir de ganglions lymphatiques situés dans le sinus rénal ou à partir du réseau lymphatique entourant la capsule rénale a été suggéré. L'inflammation chronique et les lésions répétitives du parenchyme rénal pourraient également induire le recrutement, la transformation des lymphocytes, et le développement d'un processus lymphomateux. Une association entre le lymphome rénal et d'autres maladies infectieuses et inflammatoires chroniques a été décrite. C'est le cas notamment de la maladie de Sjögren, du lupus érythémateux disséminé, de la pyélonéphrite chronique, et des infections par le virus d'Epstein Barr (EBV). Des antécédents de transplantation rénale et d'immunosuppression chronique exposent les patients à un risque supérieur de développer une tumeur maligne, survenant en particulier dans le cadre des syndromes lymphoprolifératifs posttransplantation, souvent associés à une infection par EBV [1,2,5].

Un stroma tumoral riche en lymphocytes, accompagnant un carcinome rénal doit être soigneusement analysé afin d'éliminer un éventuel processus lymphomateux. Si cet infiltrat lymphoïde s'avère être de nature lymphomateuse, et que le bilan d'extension se révèle négatif, le diagnostic d'une tumeur hybride, associant un carcinome rénal et un lymphome rénal primitif, sera retenu.

\section{Déclaration de liens d'intérêts}

Les auteurs déclarent ne pas avoir de liens d'intérêts.

\section{Références}


[1] Shetty S. Primary Renal Lymphoma - A Case Report and Review of Literature. J Clin Diagn Res 2016;10(9):05-7. https://doi.org/10.7860/JCDR/2016/20901.8577.

[2] Chen X, Hu D, Fang L, Chen Y, Che X, Tao J, et al. Primary renal lymphoma: A case report and literature review. Oncol Lett 2016;12:4001-8. https://doi.org/10.3892/ol.2016.5173.

[3] Kunthur A, Wiernik PH, Dutcher JP. Renal parenchymal tumors and lymphoma in the same patient: Case series and review of the literature. Am J Hematol 2006;81:271-80. https://doi.org/10.1002/ajh.20533.

[4] Serefhanoglu S, Buyukasik Y, Goker H, Akin SC, Akin S, Sayinalp N, et al. Concomitant renal cell carcinoma and lymphoid malignancies: a case series of five patients and review of the literature. Med Oncol 2010;27:55-8. https://doi.org/10.1007/s12032-009-9170-7.

[5] Kocher NJ, Leung S, Sarwani NE, Warrick JI, Raman JD. Synchronous Primary Renal Lymphoma and Renal Cell Carcinoma: Collision Tumors in the Same Kidney. J Endourol Case Rep 2017;3:87-9. https://doi.org/10.1089/cren.2017.0055. 